\title{
Design of Mimo-Ofdm Using Fixed Sphere Decoding Detection Method
}

\author{
K.Sathya,(M.E), S.S.Raju, M.E., Asst Professor,V. Prabu Kumar, M.E.,Asst \\ Professor, T.Shiva Prasad,M.E.,Asst Professor, \\ Veltech Multitech Dr.Rangarajan Dr.Sakunthala Engg College, Chennai.
}

\begin{abstract}
Designing MIMO-OFDM using fixed sphere decoding detection method is implemented. The design includes OFDM-Transmitter and receiver sending 4 signals from transmitter to receiver with fixed sphere decoding detection method. In this paper OFDM-Transmitter process is implemented using xilinix software. In transmitter process, includes 16 QAM,IFFT. Four signals with 16 sample values are send for transmitter. In IFFT radix 416 point is used, it reduces computational complexity. In the proposed system fixed sphere decoding detection method is used in receiver section, we can achieve a fast detection approach for OFDM receiver-will detect the received signals quickly.FSD advantages is that no of visited nodes will be same for Hard and Soft output hence they have same throughput.
\end{abstract}

\section{Introduction}

In the forth coming of next generation of wireless communication system, MIMO-Multiple Input Multiple Output will achieve high data rates for signal transmission and improve BER performance .Technique one called Maximum Likelihood (ML) in that it faces a problem of computational complexity with more no of transmit antennas and receiver antennas and this is not used efficiently in the view point of practical applications. Next technique called for MIMO detection Zero Forcing Minimum ,Mean Square and V- blast. These are also varied in point BER performance and complexity. Recent technique called Sphere Decoding(SD) uses K-best algorithm with tree based search method, achieve reduced complexity by searching only over finite no of symbols with radius of circle $\mathrm{R}$ centered at received section, here this method it is not searching all possible combinations of transmitted symbols so it reduces computational complexity. But (SD) faces noise levels and channel coefficient varied, during searching process it consumes time so speed is obtained as much as we expect. Overcome all problems here we propose a method called fixed sphere decoding (FSD) detection method it is not search very lowest tree level immediately in order to updating the radius valve, because traversal order is very flexible in the tree a pair of parent and child nodes is indepentent

\section{Literature Survey}

MLD- Maximum-like hood detector is used for MIMO detector, the complexity is high, throughput is variable, so K-BEST Algorithm is used[1] computational complexity is less expensive and high throughput Kwan-wai wong[1] 4 transmit and 4 receive antennas system using 16 QAM is used and achieved throughput of $10 \mathrm{mbits} / \mathrm{sec}[1]$. K-best schnorr-euchner(KSE) decoding algorithm[2] these method is very well suited for VLSI implementation to support soft outputs[2] Modified K-best schorr-euchner(MKSE) is used[2], these implementation achieves near ML-performance and high throughput detection with less complexity. A SoftOutput Modified Metric First (MMF)-LSD,[3]this reduces memory requirements they have used 4 transmit steam with $16 \& 64$ QAM it decrease average no of visited nodes[3] . SDM- Space Division Multiplexing[4] it reduces radius valves just by shifting centre of constellation it reduces processing time and improves frequency of operation and it core area is small not necessary to have large memory[4].K-best algorithm[5] is best suitable for higher order constellation schemes and achieved high throughput without loss of BER performance[5] it achieved SNR with independently. Systolic soft detector architecture [6]for high dimension here a single detector core will achieve a throughput of $215 \mathrm{Mbps}$.[7] it is a fully pipelined sorter generating one result per clock cycle and detection rate $1.5 \mathrm{Gbps}[7]$.

\subsection{QAM IN OFDM}

\section{Methodology}

OFDM includes Transmitter process and Receiver process section. An OFDM carrier signal is the summation number of orthogonal sub-carriers, these are independently modulated by using of quadrature amplitude modulation (QAM) . Proposed system uses QAM modulation so 16 constellation points are used. In QAM, To understand the basic idea behind the QAM approach, let $\mathrm{x}_{1}(\mathrm{t})$ and $\mathrm{x}_{2}(\mathrm{t})$ be two band limited low frequency signals eith a bandwidth of $\Omega_{\mathrm{m}}$. The two modulating signals are individually modulated by the two carrier signals $A \cos \left(\Omega_{0} t\right)$ and $A \sin \left(\Omega_{0} t\right)$, respectively, and are summed, resulting in a composite signal y(t) 
given by $y(t)=A x_{1}(t) \cos \left(\Omega_{0} t\right)+A x_{2}(t) \sin \left(\Omega_{0} t\right)$. Two carrier signals have the same carrier frequency $\Omega_{0}$ but having a phase difference of 90 degree. The carrier $A \cos \left(\Omega_{0} t\right)$ is called in-phase component and carrier $A$ $\sin \left(\Omega_{0} \mathrm{t}\right)$ is called the quadrature component. The spectrum $\mathrm{Y}(\mathrm{j} \Omega)$ of the composite signal $\mathrm{y}(\mathrm{t})$ is now given by $\mathrm{Y}(\mathrm{j} \Omega)=\mathrm{A} / 2\left\{\mathrm{X}_{1}\left(\mathrm{j}\left(\Omega-\Omega_{0}\right)\right)+\mathrm{X}_{1}\left(\mathrm{j}\left(\Omega+\Omega_{0}\right)\right)\right\}+\mathrm{A} / 2\left\{\mathrm{X}_{2}\left(\mathrm{j}\left(\Omega-\Omega_{0}\right)\right)+\mathrm{X}_{2}\left(\mathrm{j}\left(\Omega+\Omega_{0}\right)\right)\right.$ and is seen to occupy the same bandwidth as the modulated signal obtained by a DSB modulation. To recover the original modulating signals , the composite signal is multiplied by both the in-phase and the quadrature components of the carrier separately, resulting in two signals: $\mathrm{r}_{1}(\mathrm{t})=\mathrm{y}(\mathrm{t}) \cos \left(\Omega_{0} \mathrm{t}\right), \mathrm{r}_{2}(\mathrm{t})=\mathrm{y}(\mathrm{t}) \sin \left(\Omega_{0} \mathrm{t}\right)$, here four signals with each one 16 samples are given input to QAM-16. QAM 16 produces 16 real and imaginary valves for each signals.

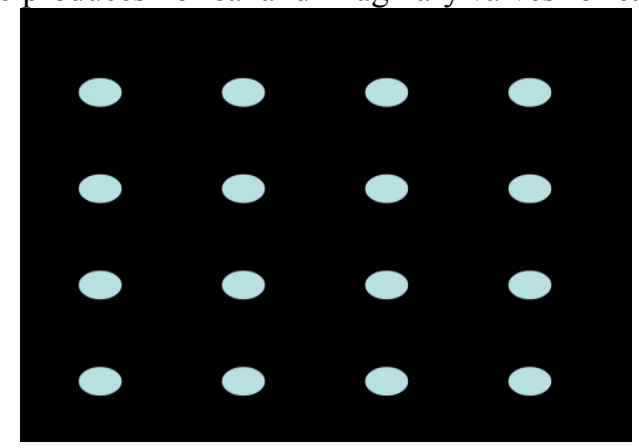

\subsection{IFFT IN OFDM}

Fig1.1 QAM-16 CONSTELLATION MAPPING

These 16 real and imaginary valves are passed to IFFT block. In IFFT radix 4 _16 point is used rather than radix 2 16 point because it reduces computational complexity. IFFT is used in OFDM transmitter process because IFFT eliminates individual multipliers and along with eliminates separate sinusoidal converters. IFFT eliminates so much of using oscillators. It only need one oscillators. Radix 2 _ 16 point uses 4 multiplier and 8 adders but radix $4 \_16$ point uses 3 multipliers and 12 adders. These 4 input signals are transmitted in the transmitter process successfully.

\section{TRANSMITTER SECTION}

RECEIVER SECTION

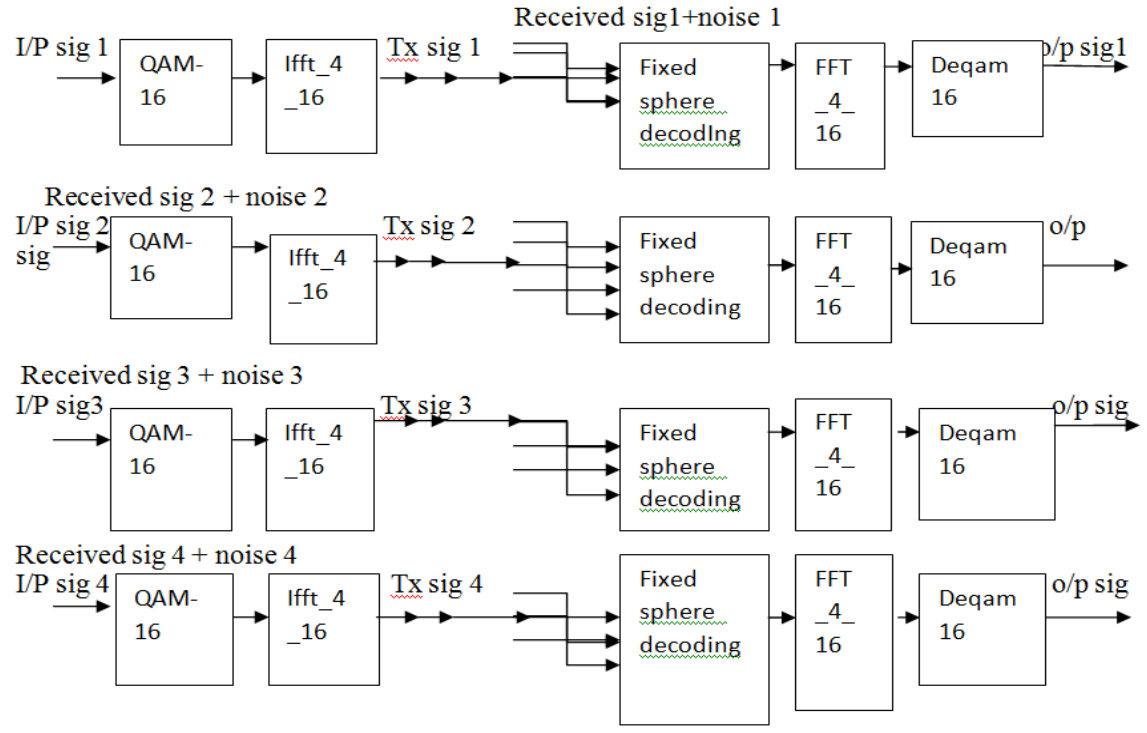

Fig 1.2 Block Diagram of Proposed System

Next in the receiver section Fixed Sphere Decoding detection method is used. Fixed Sphere Decoding, searches over a fixed number of vectors within a circle of radius $C$ centred at the received section. Since in FSD no of visited nodes will be same for Hard and Soft output hence they have same throughput.

\section{Simulation Results And Discussion}

OFDM-transmitter section process was implemented and simulated by using xilinix software. Simulation results shown in the figure 1.3 below shows that 1 signals with 16 sample value to qam input, it produces 16 real and imaginary valves obtained. Figure $1.4,1.4 .1,1.4 .2$ shows that IFFT output by Radix 4 16 point method calculated 4 signals with 16 samples with real and imaginary valves. 


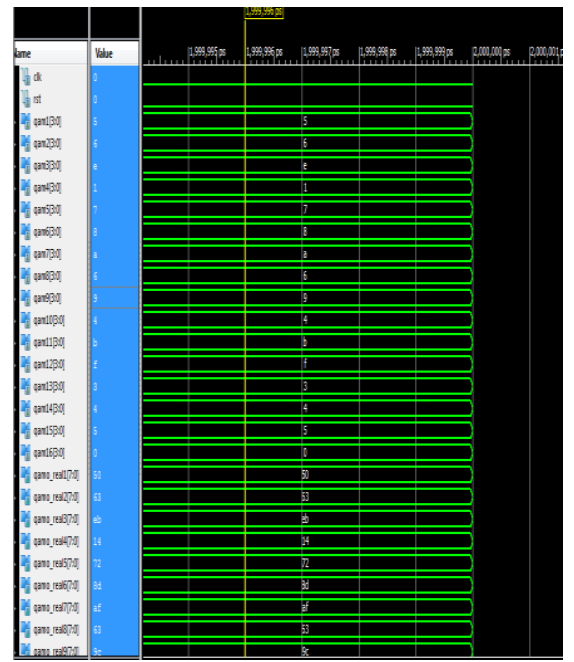

Fig 1.3 Output of QAM

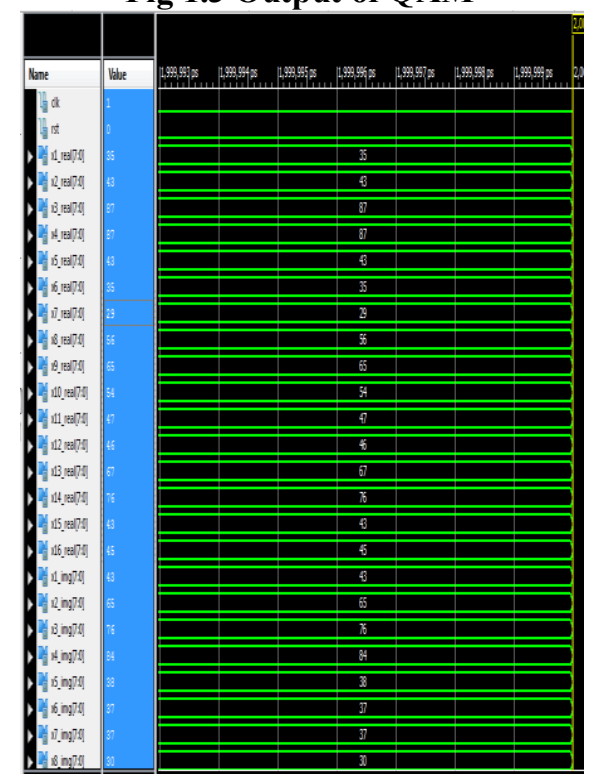

Fig 1.4 output of IFFT

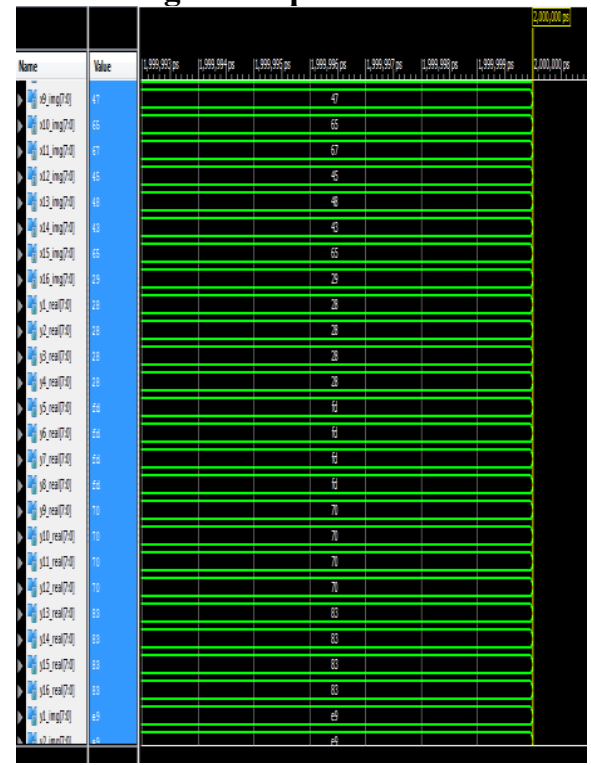

Fig 1.4.10utput of IFFT 


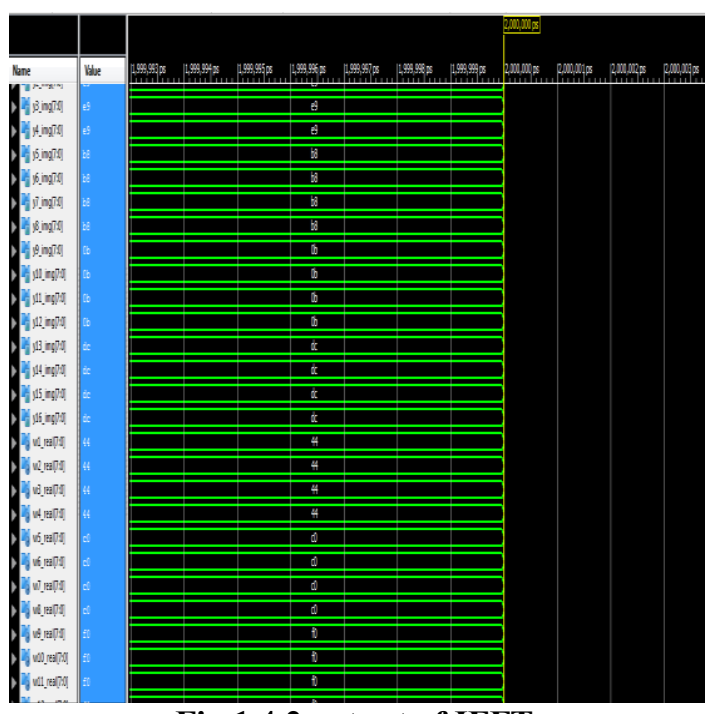

Fig 1.4.2 output of IFFT

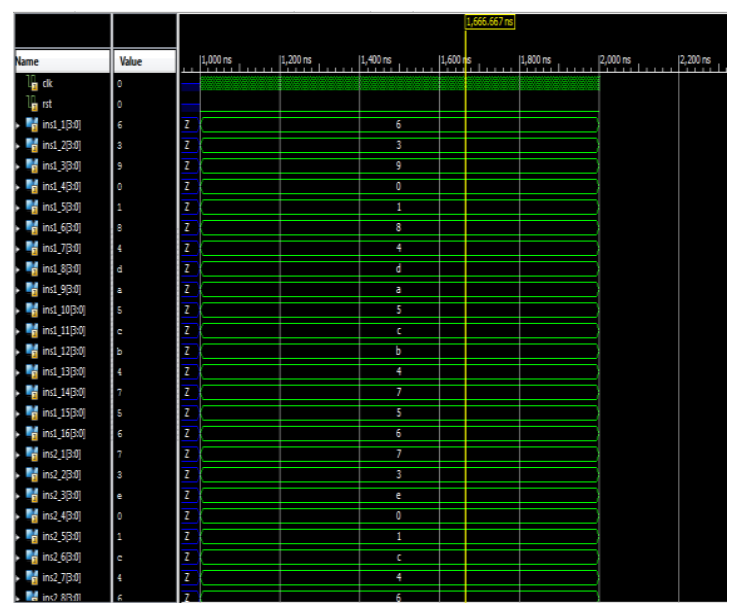

Fig 1.5 Output of Transmitter section

\section{Conclusion And Future Work}

Thus designing of MIMO-OFDM in transmitter section was implemented and simulated results shown above using xilinix software. Further receiver section will be implemented by xilinix software with a technique called Fixed Sphere Decoding Detection method.

\section{References}

[1] K. W. Wong, C. Y. Tsui, R. S. K. Cheng, and W. H. Mow, "A VLSIarchitecture of a K-best lattice decoding algorithm for MIMO channels,"'in Proc. IEEE Int. Symp. Circuits Syst., vol. 3. May 2002, pp. 273-276

[2] Z. Guo and P. Nilsson, "Algorithm and implementation of the K-best sphere decoding for MIMO detection," IEEE J. Sel. Areas Commun.,vol. 24, no. 3, pp. 491-503, Mar. 2006.”

[3] Markus Myllylä, Joseph R. Cavallaro, and Markku Juntti “ Architecture Design and Implementation of the Metric First List Sphere Detector Algorithm", may 2011

[4] H.-L. Lin, R. C. Chang, and H. Chan, "A high-speed SDM-MIMO decoder using efficient candidate searching for wireless communication,”IEEE Trans. Circuits, Syst. II, vol. 55, no. 3, pp. 289-293, Mar. 2008.

[5] M. Shabany and P. G. Gulak, "A $675 \mathrm{Mb} / \mathrm{s}, 4 \times 4$ 64-QAM K-best MIMO detector in $0.13 \mu \mathrm{m}$ CMOS," IEEE Trans. Very Large ScaleIntegr. (VLSI) Syst., vol. 20, no. 1, pp. 135-147, Jan 2012.

[6] Pankaj Bhagawat, Rajiballav Dash and Gwan Choi "Systolic Like Soft Detection Architecture for 4x4 64 -QAM MIMO System".2009

[7] Pei-Yun Tsai, Wei -Tzuo Chen, Xing-cheng Lin and Meng -Yuan Huang “A 4X4 64-qam Reduced- complexity K -best MIMO Detector upto 1.5 Gbps". 